\title{
Retenção Urinária Como Manifestação de Hematocolpos
}

\author{
Urinary Retention Due to Hematocolpos
}

Íris Santos SILVA®1, Renata MARTELLO ${ }^{1}$, António MENDES 1 , Albertino CHAVES ${ }^{2}$

Acta Med Port 2021 Mar;34(3):232-235 - https://doi.org/10.20344/amp.11912

\section{RESUMO}

O hematocolpos consiste na obstrução do fluxo menstrual por uma anomalia do trato genital, sendo a mais frequente o hímen imperfurado, uma patologia pouco comum (prevalência de 1:1000 - 1:16 000). Geralmente é uma condição assintomática até a menarca, quando ocorre acumulação de sangue na vagina (hematocolpos) ou no útero (hematometra). Um sintoma raro de hematocolpos é a retenção urinária. Apresentamos o caso de uma adolescente de 12 anos, sem menarca e com estadio sexual de Tanner M4/P5, que recorreu ao nosso serviço de urgência com dor abdominal e retenção urinária. Com o intuito de sensibilizar para esta patologia rara, fizemos uma breve revisão teórica orientada para o rápido diagnóstico e tratamento da mesma.

Palavras-chave: Adolescente; Hematocolpos; Hímen; Retenção Urinária/etiologia

\section{ABSTRACT}

Hematocolpos is an obstruction of the menstrual flow due to an anomaly of the genital tract, with imperforate hymen being the most common one. It is an uncommon condition, with a prevalence of 1:1000-1:16 000. It is usually an asymptomatic condition until the onset of menarche, when there is accumulation of blood in the vagina (hematocolpos) or in the uterus (hematometra). A rare symptom of hematocolpos could be urinary retention. Our case report is about a 12-year-old girl, with no menarche and Tanner stage M4/P5, who was admitted to our emergency department with abdominal pain and urinary retention. A brief review of this rare condition is presented, in order to remind clinicians about its existence, so that it can be diagnosed and treated as quickly as possible.

Keywords: Adolescent; Hematocolpos; Hymen; Urinary Retention/etiology

\section{INTRODUÇÃO}

As anomalias estruturais do aparelho reprodutor feminino podem-se manifestar em fases diferentes do crescimento. ${ }^{1} \mathrm{O}$ hímen é um remanescente embriológico do tecido mesodérmico, que, normalmente, perfura durante os últimos estágios do desenvolvimento embrionário. Quando imperfurado é a anomalia obstrutiva mais frequente do trato genital feminino, com uma prevalência de 1:1000 meninas recém-nascidas. ${ }^{2-6}$

Os sintomas mais comuns do hímen imperfurado são dor abdominal cíclica, dor lombar e tumefacção pélvica, geralmente entre os 13 e 15 anos de idade (quando ocorre a menarca). Apesar da inexistência de menarca, as características sexuais secundárias encontram-se habitualmente bem desenvolvidas. Como o fluxo vaginal é obstruído, o sangue menstrual acumula-se na vagina (hematocolpos) e no útero (hematometra). ${ }^{7} \mathrm{~A}$ retenção urinária aguda é uma apresentação rara de hematocolpos. ${ }^{3,8}$

\section{CASO CLÍNICO}

Adolescente de 12 anos de idade, do sexo feminino, trazida ao serviço de urgência pediátrica por quadro de retenção urinária com duração de 10 horas. Foi feita referência a dor abdominal esporádica com quatro meses de evolução, associada a obstipação e localizada aos quadrantes inferiores e região pélvica, sendo de caráter constante e agudizado no dia da vinda. Negava dor ou ardor miccional, febre, vómitos ou perda ponderal.

Como antecedentes pessoais, apresentava rinite alérgica e duas cirurgias prévias (remoção de quisto sebáceo e fibroma na língua). Não havia apresentado menarca até ao momento. Sem antecedentes familiares de relevo.

Ao exame objetivo verificou-se estadio de Tanner M4/ P5, palpação dolorosa nos quadrantes inferiores, com globo vesical. Foi algaliada e, após o esvaziamento vesical, mantinha uma ligeira massa no hipogastro, algo desconfortável à palpação.

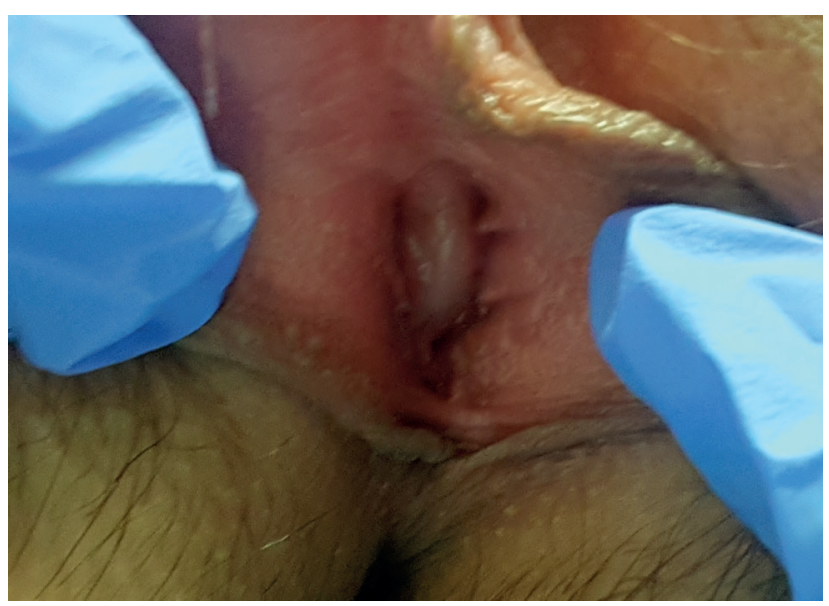

Figura 1 - Hímen imperfurado protruso

1. Serviço de Pediatria. Hospital Sousa Martins. Unidade Local de Saúde da Guarda. Guarda. Portugal.

2. Serviço de Ginecologia e Obstetrícia. Hospital Sousa Martins. Unidade Local de Saúde da Guarda. Guarda. Portugal.

$\triangle$ Autor correspondente: Íris Santos Silva. irissantossilva.92@gmail.com

Recebido: 31 de maio de 2019 - Aceite: 16 de setembro de 2019 - First published: 18 de setembro de 2020 - Online issue published: 01 de fevereiro de 2021 Copyright $\odot$ Ordem dos Médicos 2021 
Foi solicitada colaboração da Ginecologia, que confirmou a presença de hímen imperfurado (Fig. 1) e realizou estudo ecográfico, que revelou presença de massa de sangue intravaginal (hematocolpos) (Fig.s 2 e 3).

A avaliação analítica pré-operatória apresentava 18470 leucócitos/uL com $90 \%$ de neutrófilos, proteína C reativa negativa, tempos de coagulação normais e sumária de urina sem alterações. A adolescente, em contexto de urgência, foi submetida a permeabilização do hímen (himenotomia cruciforme, seguida de excisão central circular) sob anestesia geral (Fig. 4), com drenagem de cerca de 1,5 L de conteúdo hemático.

Realizou-se antibioterapia com flucloxacilina endovenosa e clindamicina tópica. Ao segundo dia de internamento, a doente mostrou boa evolução clínica, foi confirmada a permeabilidade do hímen com cotonete e não existiam sinais inflamatórios. Teve alta médica ao terceiro dia, com indicação de manutenção de clindamicina tópica por mais dois dias e flucloxacilina oral até cumprir oito dias. Os ciclos menstruais seguintes foram regulares.

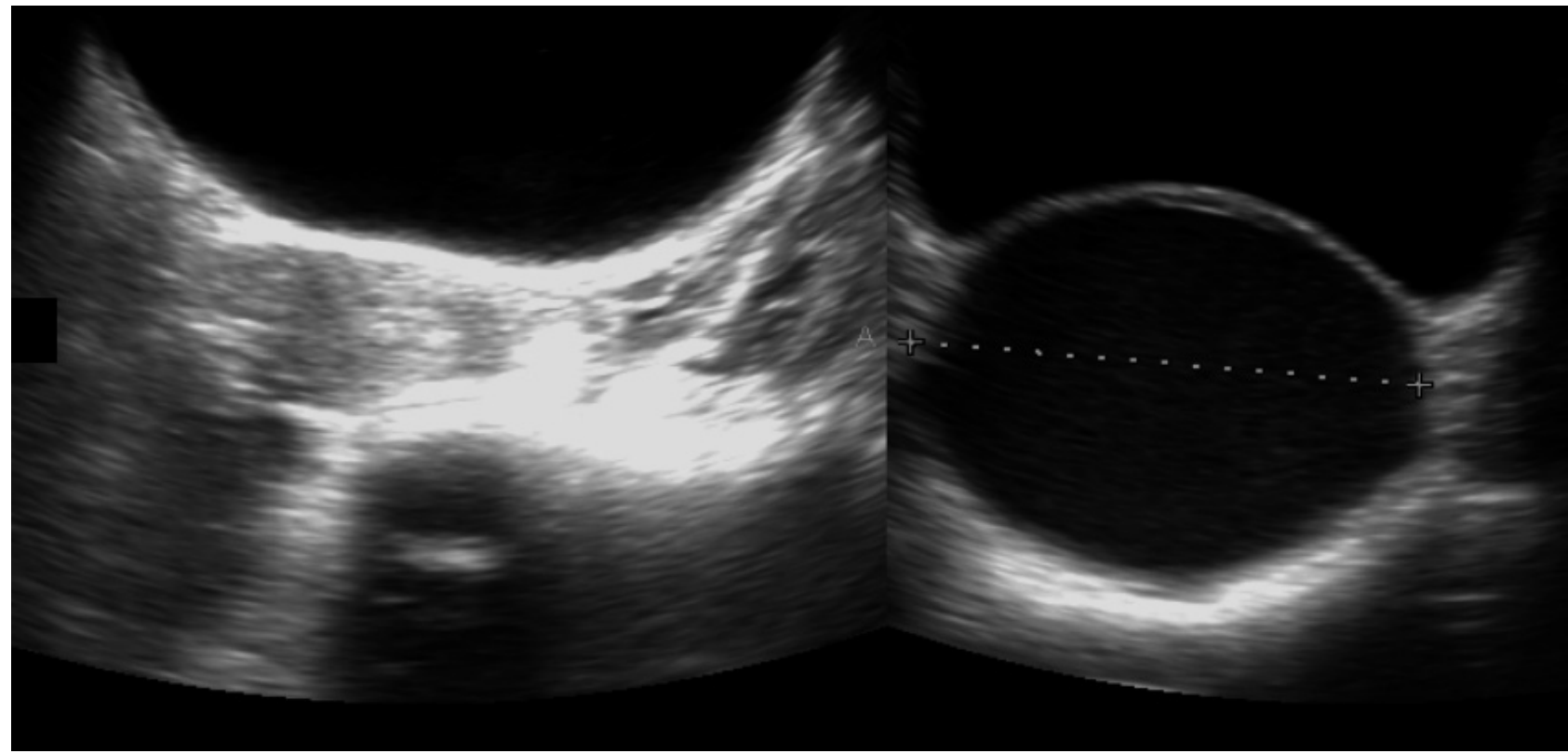

Figura 2 - Bexiga distendida e acumulação de sangue no canal vaginal

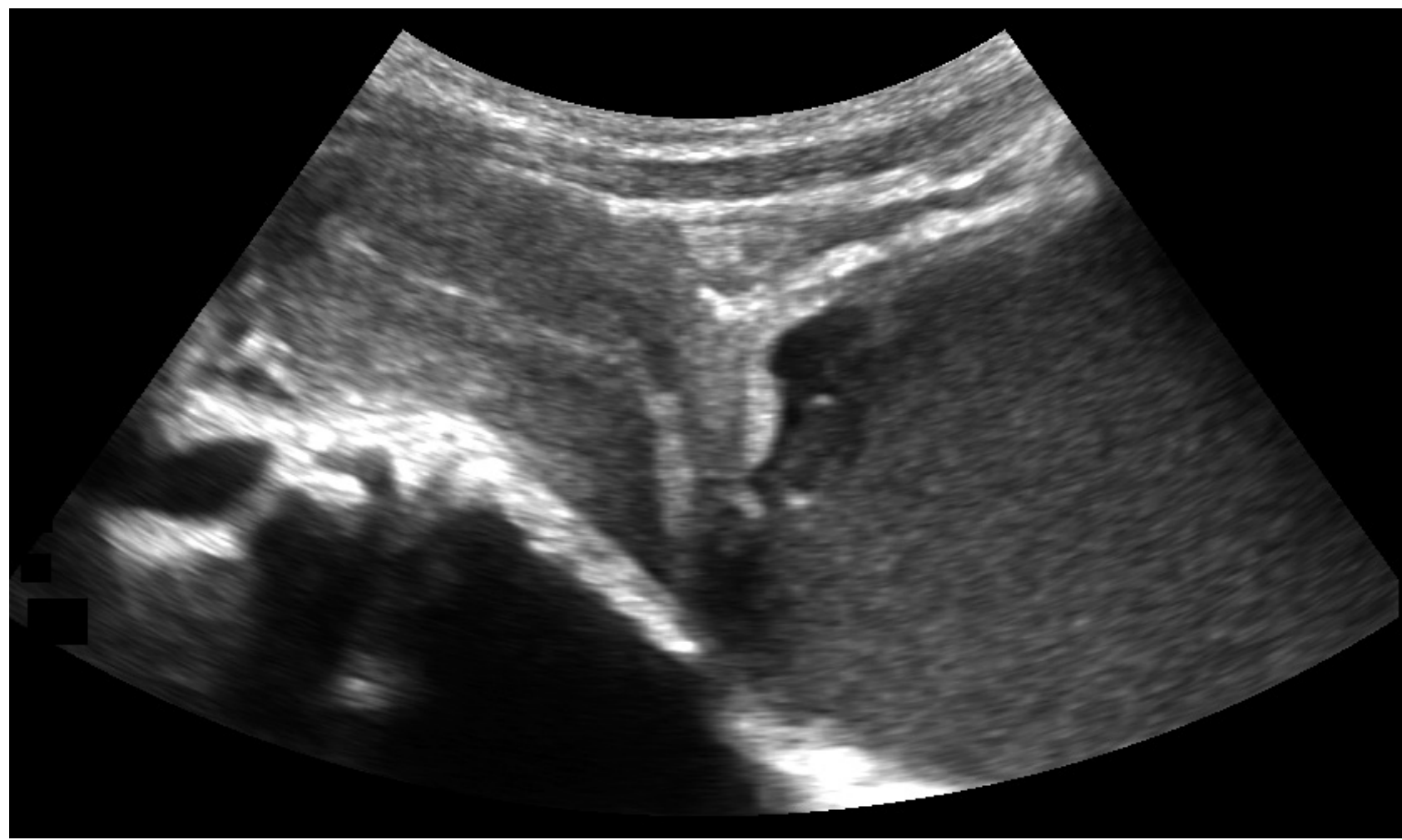

Figura 3 - Sangue acumulado no canal vaginal 


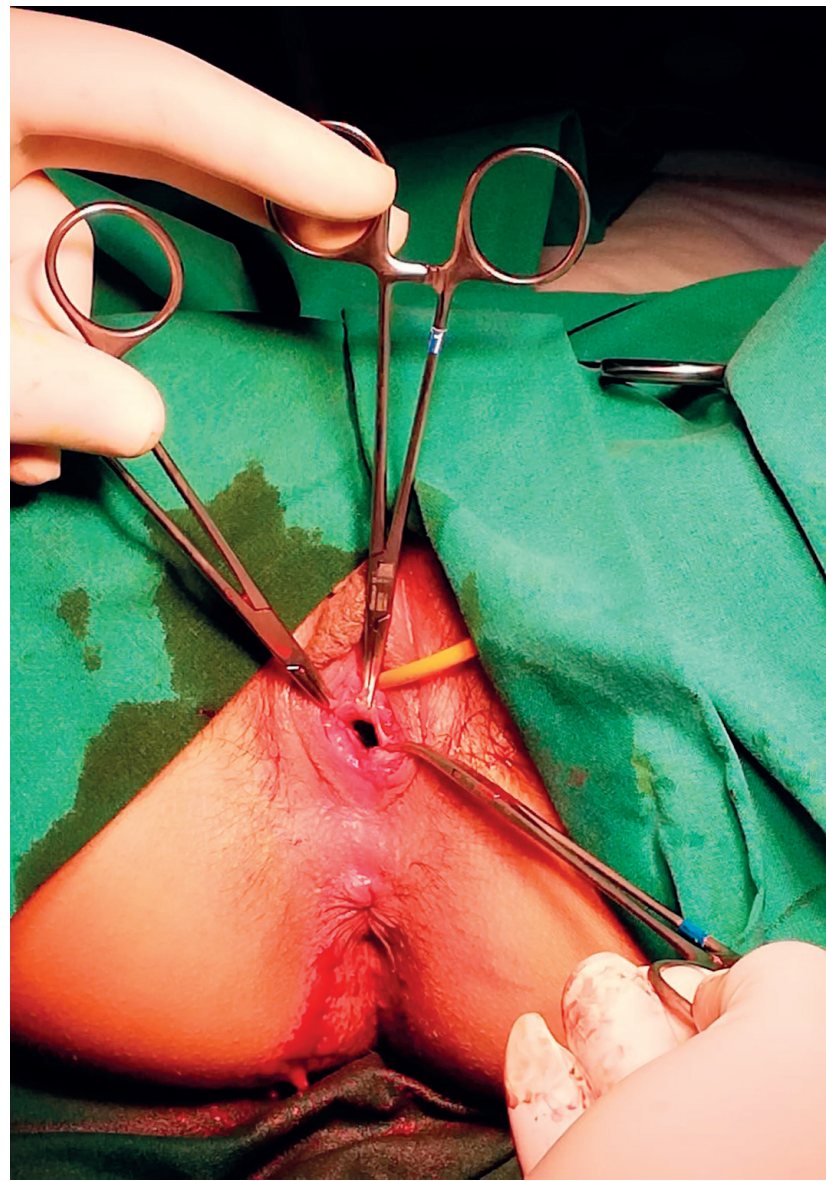

Figura 4 - Aspecto pós-operatório, após himenotomia

\section{DISCUSSÃO}

O hímen é uma membrana que se desenvolve embriologicamente através da fusão da extremidade caudal dos ductos paramesonéfricos e do seio urogenital. A porção central desta membrana perfura através da degeneração das suas células epiteliais. A falha da degeneração, e subsequente perfuração, dessas células leva a um hímen denominado imperfurado, ${ }^{2}$ geralmente, diagnosticado nos recém-nascidos ou, posteriormente, na adolescência. Numa adolescente com dor abdominal cíclica, sintomas de pressão e, frequentemente, massa abdominal e/ou pélvica, podemos estar perante um hematocolpos. Esta é uma condição rara, causada pela obstrução do fluxo sanguíneo menstrual, devido a uma anomalia obstrutiva do trato genital. ${ }^{1,3,8}$

A causa mais comum é o hímen imperfurado, com uma incidência muito baixa, de 1:1000 a 1:16 000 mulheres, embora os dados epidemiológicos sejam escassos. McCann et al relataram uma incidência de $1,2 \%(n=114)$ num estudo descritivo sobre achados genitais em meninas com idades entre 10 meses e 10 anos de idade, e Evans et al encontraram uma taxa de $6,7 \%$ de hímen imperfurado $(n=254)$ no seu trabalho sobre malformações vaginais. Normalmente, esta anomalia ocorre de forma isolada, embora, em alguns casos, ocorrências familiares ou hereditárias tenham sido relatadas. ${ }^{7-9}$ Além disso, a ocorrência de anomalias combinadas (por exemplo, hímen imperfurado e septo vaginal transverso), embora seja rara, já foi relatada, sendo portanto necessária avaliação pré-operatória cuidadosa para exclusão dos septos vaginais transversos. ${ }^{3}$

O estudo ecográfico é o exame de escolha para a maioria dos casos de hímen imperfurado com hematocolpos e hematometra relatados na literatura. Este é importante não apenas para confirmar a ocorrência de hematocolopos, mas também da causa subjacente, através da diferenciação entre hímen imperfurado ou septo vaginal. ${ }^{11}$ Esta realiza-se através da manobra de Valsalva: um hímen imperfurado deve ser protuberante, ao contrário do que ocorre com o septo vaginal transverso. ${ }^{7}$ A ecografia exclui ainda a ocorrência de outras malformações mullerianas ou anormalidades urológicas, que podem estar relacionadas com estas em $25 \%$ a $90 \%$ das mulheres. ${ }^{9}$ Apesar da importância da ecografia nestas situações, muitas vezes, recorre-se à ressonância magnética para uma avaliação complementar, sobretudo se houver suspeita de malformação uterina obstrutiva alta. ${ }^{1}$ A retenção urinária aguda, verificada nesta doente, é uma apresentação muito rara de hematocolpos, com poucos casos relatados na literatura, ${ }^{8}$ devendo-se aos efeitos mecânicos exercidos pelo fluxo menstrual obstruído sobre a bexiga e a uretra. ${ }^{10}$ Pelo mesmo efeito de compressão, outros sintomas que podem estar presentes são a obstipação, o edema dos membros inferiores, e a dor lombar, devido à irritação do plexo sagrado ou das raízes nervosas. $^{7}$

O diagnóstico precoce de um hímen imperfurado é importante, pois esta situação clínica pode levar a complicações graves, nomeadamente infecções, hidronefrose, insuficiência renal, endometriose e subfertilidade..$^{2,7} \mathrm{O}$ tratamento é destinado a restabelecer o fluxo vaginal e consiste em himenotomia sob anestesia local ou geral. Podem ser utilizadas incisões simples verticais, em forma de T, ou cruciformes, em forma de X. A técnica utilizada foi incisão, em forma de $X$, central circular. Optou-se por fazer uma incisão cruciforme, uma vez que tem a vantagem de reduzir o risco de lesão na uretra, seguida de uma excisão central circular para manter um hímen anular intacto. 6,11 A fixação da mucosa vaginal ao intróito evita a reestenose e resulta em pós-operatório sem complicações. ${ }^{2,5}$ Contudo, neste caso, devido à espessura do hímen, optou-se por não se realizar a fixação.

O hematocolpos, apesar de raro, é uma causa de dor abdominal e retenção urinária em adolescentes, de modo que se deve levantar a suspeita quando a estas queixas se associa a ausência de menarca.

\section{PROTEÇÃO DE PESSOAS E ANIMAIS}

Os autores declaram que os procedimentos seguidos estavam de acordo com os regulamentos estabelecidos pelos responsáveis da Comissão de Investigação Clínica e Ética e de acordo com a Declaração de Helsínquia da Associação Médica Mundial publicada em 2013.

\section{CONFIDENCIALIDADE DOS DADOS}

Os autores declaram ter seguido os protocolos do seu 
centro de trabalho acerca da publicação de dados.

\section{CONSENTIMENTO DO DOENTE}

Obtido.

\section{CONFLITOS DE INTERESSE}

Os autores declaram não ter conflitos de interesses relacionados com o presente trabalho.

\section{REFERÊNCIAS}

1. Kachhawa G, Kriplani A. Management of reproductive tract anomalies. J Obstet Gynecol India. 2017;67:162-7.

2. Mwenda AS. Imperforate hymen - a care cause of acute abdominal pain and tenesmus: case report and review of the literature. Pan Afr Med J. 2013;15:28.

3. Khemchandani S, Devra A, Gupta S. An unusual case of urinary tract obstruction due to imperforate hymen in an 11-month-old infant. Indian J Urol. 2007;23:198-9.

4. Silva S, Almeida S. Causa rara de dor abdominal numa adolescente. Acta Pediatr Port. 2008;39:216-8.

5. Laghzaoui O. Congenital imperforate hymen. BMJ Case Rep. 2016;2016:bcr2016215124.

6. Temizkan O, Kucur SK, Ağar S, Gözükara I, Akyol A, Davas I. Virginity sparing surgery for imperforate hymen: report of two cases and review of literature. J Turk Ger Gynecol Assoc. 2012;13:278-80.

\section{FONTES DE FINANCIAMENTO}

Este trabalho não recebeu qualquer tipo de suporte financeiro de nenhuma entidade no domínio público ou privado.

7. Lardenoije C, Aardenburg R, Mertens H. Imperforate hymen: a cause of abdominal pain in female adolescents. BMJ Case Rep. 2009;2009:bcr08.2008.0722.

8. Patoulias I, Prodromou K, Kallergis K, Koutsoumis G. Acute urinary retention due to hematocolpos: report of two cases. J Pediatr Surg Case Rep. 2013;1:189-91.

9. Chircop R. A case of retention of urine and haematocolpometra. Eur J Emerg Med. 2003;10:244-5.

10. Chang JW, Yang LY, Wang HH, Wang JK, Tiu CM. Acute urinary retention as the presentation of imperforate hymen. J Chin Med Assoc. 2007;70:559-61.

11. Egbe TO, Kobenge FM, Wankie EM. Virginity-sparing management of hematocolpos with imperforate hymen: case report and literature review. JCMS Case Rep. 2019;7:2050313X19846765. 